CLAWAR 2018: 21st International Conference on Climbing and Walking Robots and the Support Technologies for Mobile Machines, Panama City, Panama, 10-12 September 2018

\title{
MOORING CHAIN CLIMBING ROBOT FOR NDT INSPECTION APPLICATIONS
}

\author{
MATTHEW KIMBALL, AMIT, ARTUR GMEREK, PETER COLLINS, ANDREW \\ WHEATLEY, KIRAN SHAH, JIANWEI LIU, MAHESH DISSANAYAKE ${ }^{1}$, JESSICA \\ CAROLL, ANGELOS PLASTROPOULOS, PANAGIOTIS KARFAKIS, \\ GURVINDER S VIRK AND TARIQ SATTAR ${ }^{1}$ \\ InnotecUK, Hildersham Road, Cambridge, CB21 6DR, UK \\ ${ }^{1}$ London South Bank Innovation Centre,103 Borough Road, London SE1 OAA, UK
}

\begin{abstract}
Inspection of mooring chains is an important but dangerous and costly procedure covering inspection above and below the waterline. The paper presents initial results from the RIMCAW project which aimed at designing and building an inspection robot able to climb mooring chains and deploy NDT technologies for scanning individual links thereby detecting critical defects. The paper focuses on the design and realisation of the inchworm type novel crawler developed and tested in the TWI Middlesbrough, UK water tank.
\end{abstract}

\section{Introduction}

\subsection{Background}

With increasing global energy demand, the number of floating oil and gas production systems have increased dramatically since the 1990s. Mooring chains are safety-critical structural components used to secure the Floating Production, Storage and Offloading (FPSO) systems to the seabed. These chains are subjected to extreme loading and complex twisting motions which can lead to catastrophic failures. Moreover, these systems experience high tidal waves, storms and other harsh environmental conditions. The overall structural integrity of a chain is compromised by the presence and propagation of cracks, and hence it is vital to regularly inspect them to detect the presence of defects while they are still treatable. A single mooring line failure can cost £2-10.5M [1] and can result in vessel drift, riser rupture, production shutdown and hydrocarbon release leading to major environmental problems. During 2001 to 2011, there were more than twenty mooring incidents recorded for production vessels moored for prolonged durations (15-25 years) [2]. Among this, there were 8 multiple mooring chain breaking incidents, one of which was at Maersk Oil operated "Gryphon Alpha FPSO”. After a severe storm in 2011, Gryphon broke four anchor chains and drifted off station causing considerable damage to herself and the subsea 
extraction system. The repair and upgrading work cost $\$ 1.8$ billion and took two years before regular operations could resume [3]. This reinforces the need to inspect mooring chains on a regular basis; however, this is not a trivial task. Systematically taking mooring chains out of commission for dry dock inspections is an exhausting, lengthy and costly process. While using divers to perform inspections is impractical and raises several health and safety concerns. Given these issues, the RIMCAW project was formulated to develop an automated solution where a mobile crawler robot was able to climb on mooring chains while in service and be able to perform in-situ inspection of individual chain links in both air and water.

\subsection{Review of mooring chain inspection systems}

The development of an effective chain climbing robot mechanism has been the subject of much research in recent years. However, due the mechanical complexity of climbing mooring chains, a commercially viable product has not been realised and research has not reached beyond the experimentation phase. The MoorInspect project developed a platform (shown in Figure 1a) that used an inchworm climbing locomotion method to traverse a chain. This system, weighing approximately $450 \mathrm{~kg}$ in air and carrying a 300kg NDT payload, uses two grippers to hold and lift the platform between links [4]. The ChainTest project, Figure 1b, originally proposed using a system motorised wheel climb of the links, however, due to concerns regarding the frictionless surface conditions of an in-service chain, the design shifted towards a winch-based system [5].

These previous prototype systems are heavy, cumbersome platforms that would be difficult to deploy in an actual real-world environment. Both suffer from inadequate manoeuvrability required to handle the complex geometries of mooring chains, how the chain links could be mis-aligned and the catenary curves that can arise in practise. A tracked wheel crawler robot, Figure 1c, uses a system of tracked wheels and magnetic adhesion to move along misaligned chain links [6]. While preliminary testing yielded positive results, the robot required further work to prevent slippage under loading and during the transitions between links [7], [8]. 


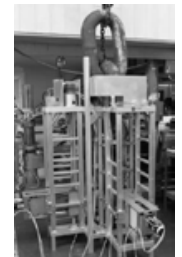

a. MoorInspect

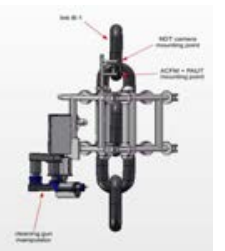

b. ChainTest

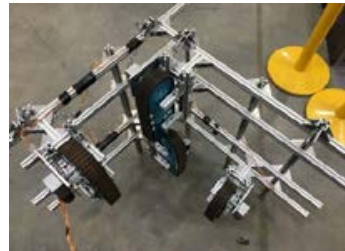

c. Track Chain Crawler

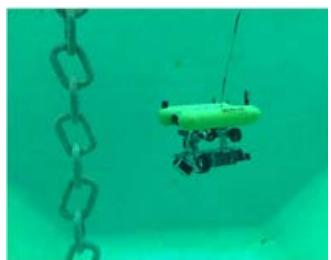

d. PANDORA AUV

Figure 1 - Mooring chain inspection systems

The "PANDORA" project, Figure 1d, aimed to produce a system to conduct chain cleaning and inspection tasks using an autonomous underwater vehicle (AUV). A high-resolution imaging sonar was used to deliver acoustic images to assist in the waypoint navigation of the robot. While water tank experiments demonstrated promising results, further refinement of the perception, planning and control algorithm was required to handle turbid water conditions [9].

\subsection{Design requirements}

Following detailed analysis, it was concluded that a compact climbing robot able to work in realistic environments needed to be developed for the solution to have any commercial viability. Such a platform must be equipped to tolerate real-world in-situ conditions, for example, able to operate in normal environmental influences (wind, tidal waves, and currents), on chain structures and condition found in current use (rusted, uneven surfaces). Furthermore, it was clear that the robot must be able to handle climbing on a range of chain scenarios and sizes as shown in Figure 2.
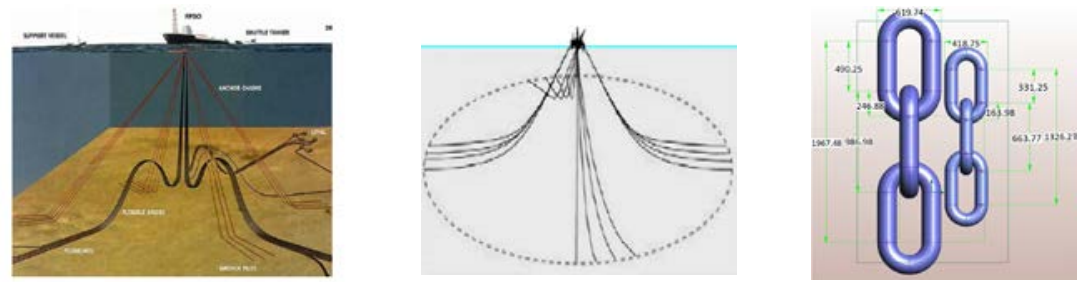

a. Taut-leg scenario in deep water b. Catenary chains in shallow water c. Mooring chains range Figure 2 - Mooring chain scenarios and range of sizes

The robot should be easily deployed and retrieved. Therefore, it is necessary to make the system lightweight while still able to carry a payload of $\approx 12 \mathrm{~kg}$ (representing a typical NDT system). The adhesion mechanism for vertical climbing should be sufficient to keep the robot attached to the chain during motion. Moreover, due to the amphibious nature of mooring chains, the developed robot must be marinized to operate in real-world normal and abnormal conditions. 


\section{Design methodology}

\subsection{Deployment plan}

Methods to deploy the robot were studied in detail to identify all procedural requirements as testing of the developed platform was planned to take place in a $7 \mathrm{~m}$ deep test tank at TWI Middlesbrough, UK with a 10-link chain suspended from an overhead gantry system into the water. The dimensions of the chain are outlined in the Figure 3.

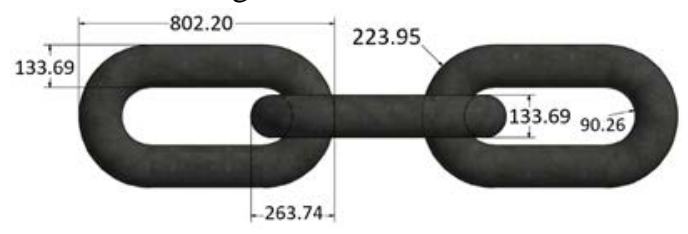

a. CAD model of test mooring chain with dimensions

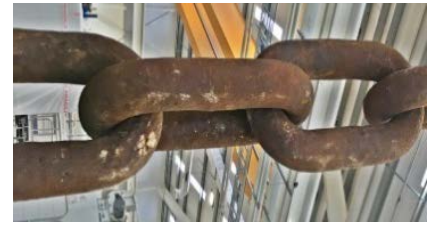

b. Picture of the testing chain Figure 3 - Details of test sample mooring chain

It was important that the prototype RIMCAW system realised was able to satisfy all the testing requirements planned and of a real deployment scenario as presented in Figure 4.

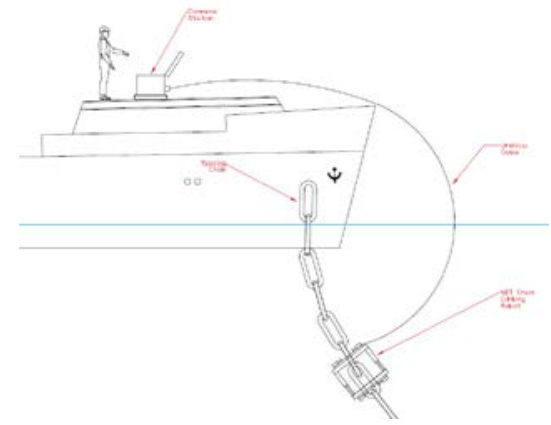

a. Real-world subsea RIMCAW scenario

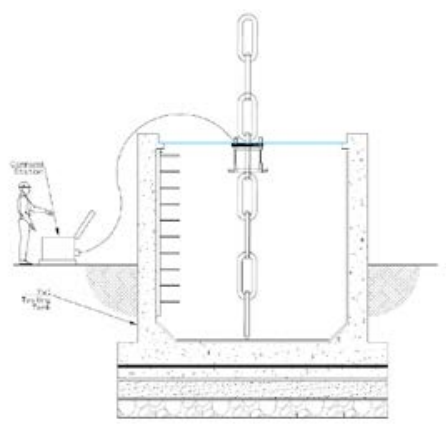

b. The tank facility testing scenario at TWI

Figure 4 - Deployment RIMCAW scenarios

\subsection{Concept design and analysis}

Five different design concepts were developed, as presented in Figure 5 with different locomotion and adhesion/gripping mechanism configurations being explored from which different crawler ideas emerged. Each design was analysed and investigated with respect to the outlined crawler criteria, climbing strategy and the associated engineering challenges. 
Concept One shown in Figure 5a, consists of three linear actuators connected between the top and bottom rings via ball bearings used to achieve the crawling motion and adaption to the catenary curve. The top ring rotates the structure around the chain, once the first set of fixed grippers have engaged with the chain link, to position the second set of grippers to grab the next link. Concept Two shown in Figure 5b, has a hinged modular double square rigid external frame connected by four differential car jack extension mechanisms with leadscrews used to perform the crawling. Both the top and bottom frames have push leadscrew actuators on each side, to grab the link from all four sides.

Concept Three shown in Figure 5c, designed to be made of modular-aluminium extrusions, is similar to Concept Two. The hinged frame and the gripper car jack leadscrew design is the same. However, the crawling is accomplished using four lead screws connected in between the top and bottom gripping structure via ball bearings. Concept Four shown in Figure 5d, also designed to be made of modularaluminium extrusions, has a hinged rectangular frame and four lead screws actuators configured in the same manner as Concept Three. The top gripper uses linear actuators that are connected to a rotary ring. The bottom gripper uses fixed lead screw jack grippers.

Concept Five shown in Figure 5e, the structure is very similar to that in Concept 1 but instead consists of four lead screws connected via ball bearings to the top and bottom frames. The top frames can rotate against each other by using a rotary ring actuator composed of a rack and pinion mechanism that allows the robot to adjust to different tilting angles of the chain elements. Two actuators are attached to each frame that is responsible for gripping the robot onto the chain. The Concept 5 design was selected after a detailed Delphi study involving the full technical team revealing that it was best suited to meet all the technical, fiscal and time requirements. During this process, Concepts 2, 3 and 4 were rejected due to the unnecessary mechanical complexity of the jack systems. Concept 1 was discarded due to its limited ability to grasp different sized chains.

However, during the early stages of the detailed design process simulation of the kinematics of the system revealed that the four-lead screw configuration would not provide the freedom required to traverse the catenary curve. Therefore, the design had to be modified slightly, and inspiration was taken from Concept 1, the linear actuator and ball joint configuration. To simplify the control and maintain 
rigidity of the platform after being split into two sections, six actuators were determined to be necessary instead of three.

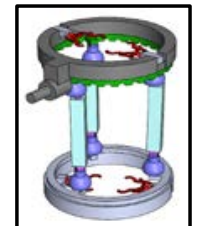

a. Concept 1

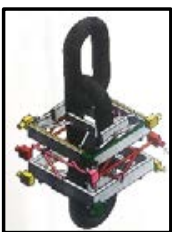

b. Concept 2

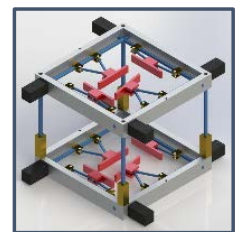

c. Concept 3

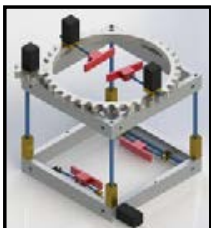

d. Concept 4

Figure 5 - RIMCAW concept designs developed and investigated

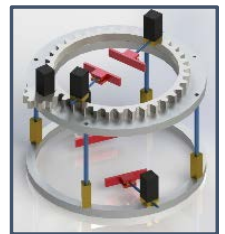

e. Concept 5

\section{RIMCAW robotic platform}

\subsection{Mechanical design}

The final RIMCAW robot design and the actual robot are shown in Figure 6; this was based on a standard Stewart platform, comprising six prismatic actuators attached in pairs to three positions on the platform's baseplate, crossing over to three mounting points on the top plate. This design allows the robot to control the pitch, roll and yaw of the top and bottom baseplates. This controllability of the platform's crawling orientation is crucial as it needs the robot to traverse the various catenary curves of mooring chains likely to be found in real scenarios. In addition, this configuration allows the robot to contract and expand allowing it to crawl by inch worming along the chain links. The method of positioning and securing the robot onto the mooring chain structure had been extensively studied and a latch/ hinge system was incorporated into the design to allow simple opening of the simplify the deployment process for the end user. A passive buoyancy system was employed to counteract the weight of the platform while in water. Circular sections of marine grade buoyancy foam were cut using CNC machining to sizes that could be inserted into the outside of the top and bottom rings.

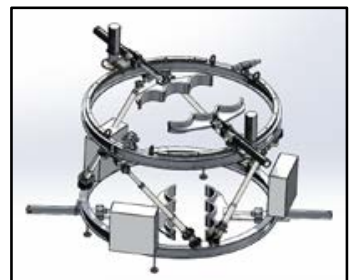

a. Final detailed CAD model

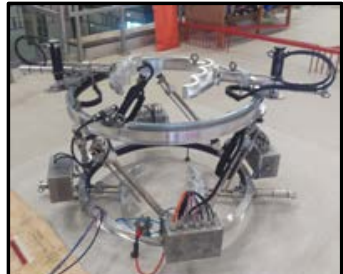

b. Actual RIMCAW robot

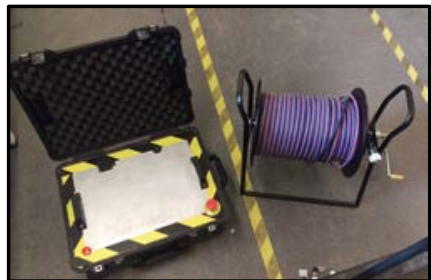

c. Command station and umbilical

Figure 6 - Detailed mechanical design 


\subsection{Electrical design}

For testing and trialling, a custom designed command and control box was required to supply power to the RIMCAW robot as shown in Figure 10. After researching British Standards for safe operating electrical power supplies in a submerged water environment, it was found that the 30V DC was the permissible SELV (Safety Extra Low Voltage) for 'Swimming Pools and Basins' outlined in BS EN 7671:2008, Regulation 702.410.3.4.1. Four IP68 enclosures with dimensions 260x160x120 mm were used to house all electronics. To minimise cables and entries, the electronics were divided into 4 sub-sections housed in different boxes. Box 1 housed the electronics required for the Stewart platform, Box 2 housed the electronics required for the gripper, Box 3 housed all the power electronics, and Box 4 housed the communications sub-systems.

\subsection{Software design}

The control of the low-level systems (actuators and sensors) was accomplished via specialised local controllers. The mid-level systems (Raspberry Pi and Arduinos) were used to coordinate the control functions specified by the top-level interface. The top-level system (GUI) provides operators with a simple means of controlling the chain crawling functionality using visual feedback from the onboard cameras.

As development progressed, the software architecture evolved, and the messaging protocol and variable details were finalised. The robot's roll $(\varphi)$, pitch $(\omega)$, yaw $(\psi)$, crawling motion, upper and lower grippers and gripper rotation could all be controlled via one message sent from either the keyboard, joystick or GUI interfacing devices. A ROS switch node was developed to provide an easy means of switching between the different forms of top level control.

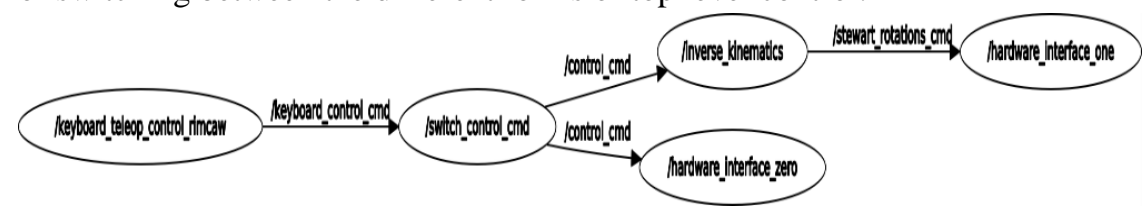

Figure 7 - ROS node/ topic network

Figure 7 shows the ROS node and topic communication structure with keyboard control outlined. The switch node passes the variable information via the ROS topic control_cmd to the gripper and Stewart platform hardware interface. The Stewart platform orientation variables (x, y z, $\varphi, \omega, \psi)$ are first converted and scaled to linear actuator positions before being passed to the hardware node. This 
translation is achieved by utilising the inverse kinematics of the robot and a separate ROS node, inverse_kinematics, was developed to perform the conversion.

\subsection{Inverse kinematics}

The kinematics of the Stewart platform is a well-documented problem and used to control the 6 actuators as required. The desired orientation of the platform requires all the linear actuators to move synchronously to different stroke positions. These positions are calculated by using the following formula and inputting the desired Cartesian position, orientation and robot joint parameters.

$$
l_{i}=T+R_{B} \cdot p_{i}-b_{i}
$$

where $T$ is the translation matrix, which describes the desired Cartesian coordinates of the top platform. $R_{B}$ is the single rotational matrix, detailing the desired extrinsic rotations around axes. It is derived from the product of the three elemental rotational matrices and the desired Euler angles.

$$
R_{B}=R_{z}(\psi) \cdot R_{y}(\omega) \cdot R_{x}(\varphi)
$$

The formula below is the expanded form of the rotational matrix with Tait-Bryan Angle Convention (Z, Y, X).

$$
R_{B}=\left(\begin{array}{ccc}
\cos \psi \cos \omega & -\sin \psi \cos \varphi+\cos \psi \sin \omega \sin \varphi & \sin \psi \sin \varphi+\cos \psi \sin \omega \cos \varphi \\
\sin \psi \cos \omega & \cos \psi \cos \varphi+\sin \psi \sin \omega \sin \varphi & -\cos \psi \sin \varphi+\sin \psi \sin \omega \cos \varphi \\
-\sin \omega & \cos \omega \sin \varphi & \cos \omega \cos \varphi
\end{array}\right)
$$

The $b_{i}$ and $p_{i}$ matrices contain the base and platform joint coordinates, respectively. These coordinates were calculated and measured using the CAD model of the designed RIMCAW platform. The height value between the base and the top platform is very important and is added to the translation matrix $\mathrm{z}$ value. It sets the minimum position the platform can move to by offsetting the $\mathrm{z}$ origin from the base to the platform. Finally, the length of the contracted actuator had to be subtracted from the calculated lengths of the kinematic formula. The remaining value then had to be scaled to a value between 0-255 for the PWM output, which translates to a stroke position on the linear actuator.

During development, a separate python script was created for testing purposes to confirm the correct operation of the kinematics. An emergency stop button was hard-wired and daisy chained to the fault pins on the linear actuator controllers. This was a means of shutting down the entire system if the inverse kinematics drove the platform into a position or workspace that could damage the hardware. Limitations of the robot are important and must be factored into the derivation of 
the inverse kinematics. There are three main areas for consideration, singularities, mechanical constraints and reachable workspace.

In robotics, singularities can pose serious problems to the overall operation and hence proximity to such singularity points needs to be monitored and avoided. To mitigate this issue, precautions had to be built into the control system where the inverse kinematics node, motions and orientations were restricted. The desired roll, pitch and yaw angles were limited to $\pm 10^{\circ}$ range and the $\mathrm{x}$, y positions were locked to zero, allowing movement in the z-axis only for performing the climbing function. For the chain climbing function, movement outside this constraint is not felt to be necessary at the initial stages. If the inverse kinematic solver derived any extension outside of its defined boundaries, the move request would be ignored, and the previous orientation would not change.

\subsection{Climbing strategy for the RIMCAW robot}

The RIMCAW platform was developed to perform a set of sequential actions to achieve the inch worming type of locomotion required to traverse a mooring chain. The robot was built to accommodate different chain dimensions by attaching specific size grippers. This would not affect the set of climbing actions, however, more or fewer steps may be required to travel the same amount of links. Figure 8 illustrates the climbing process of the platform on the test chain.

Starting with the bottom grippers extended and secured to the chain link, the top grippers are fully retracted to a safe position. The Stewart platform is then extended and orientated until the top grippers are perpendicular to the next desired chain link. This action of ensuring the correct orientation of the grippers is crucial to adapting to the catenary curve of the chain. Once in the desired position, the top grippers are extended. Upon contact with the chain, the force exerted causes the rotary ring to self-align with the link. This can only be achieved when the rotary motors are deactivated and back-drivable. With the top grippers engaged, the lower grippers are released before the Stewart platform is retracted to the grasping position of the next link. The entire platform is then rotated around the chain by $90^{\circ}$ via the actuated top gripper rotary system. The orientation of the bottom grippers, as with the top, are perpendicularly aligned with the chain before grasping the link. With the bottom gripper engaged, the process is repeated. 


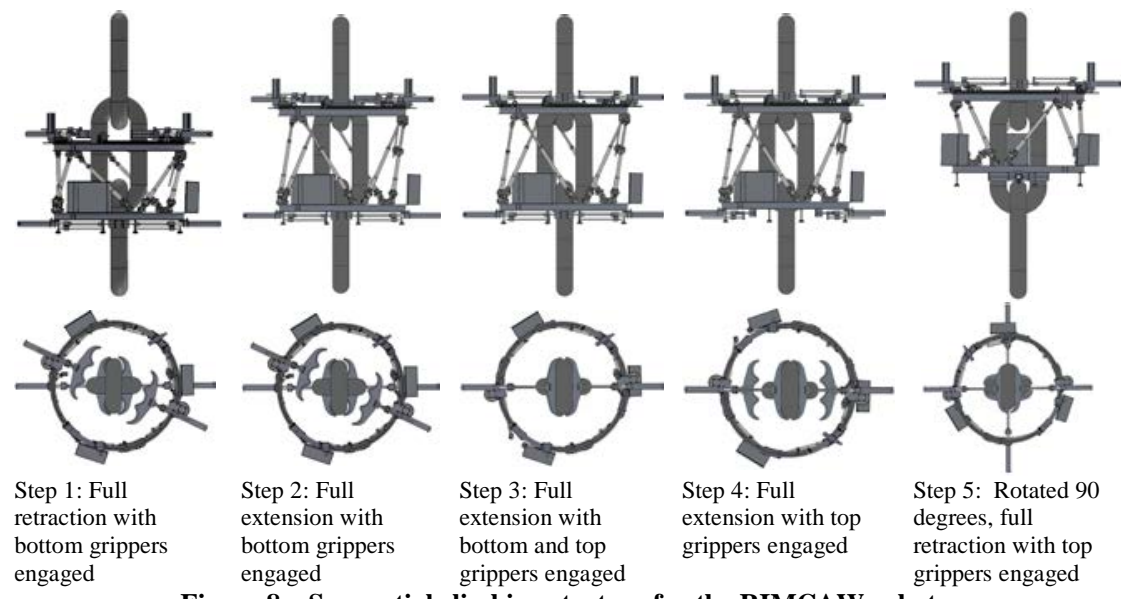

Figure 8 - Sequential climbing strategy for the RIMCAW robot

\subsection{RIMCAW control system}

Due to the time limitations of the project, a sophisticated control system was not completed, and the robot could only be driven by an operator via the command station. However, during the design and development phase, a strategy was devised to move towards autonomous climbing capability. Here a user would need only to specify a depth or position on the chain and the robot would climb unaided to the specified destination. With this defined goal, all the relevant subsystem designs were developed to accommodate this desired autonomous functionality but also meet the basic tele-operational needs.

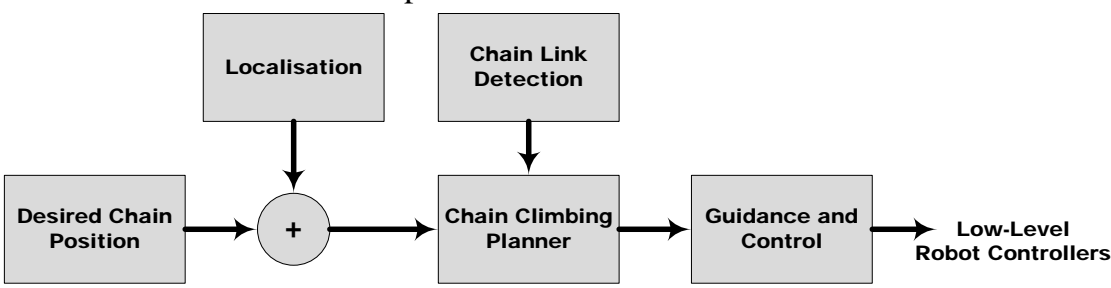

Figure 9 - Autonomous RIMCAW chain climbing framework

Figure 9 outlines the developed autonomous chain climbing framework for the RIMCAW robot. The user specified position is combined with the robot's localisation system to determine the overall travel distance. Due to the structured environment, it is not necessary to use classic position sensors on the actuators responsible for grabbing the chain and moving along it. Therefore, depth sensors were proposed as a means of approximating the position of the robot on the chain 
with respect to the FPSO. The chain link detection system builds a map of the chain geometry. Using a sonar array with equidistantly spaced sensors around the upper and lower frames, a 3D image of the chain would be compiled. From this the chain geometry could be derived using image processing and machine learning techniques as demonstrated by the perception and planning work developed in [9].

The climbing planner uses the processed geometry data from the chain link recognition system to determine the correct gripping position, orientation and climbing actions. The guidance and control systems provides the coordination of the operations required to actuate the robot's subsystems to achieve the desired objectives. The lower level controllers would be responsible for basic functional tasks such as control of individual actuators with sensory feedback. For example, the gripping system information regarding whether the robot is holding the chain would be estimated by monitoring the force exerted by the actuator. To accomplish this, the current consumption of each electric actuator would be monitored via the lower level controllers and correlated to force.

\section{Experimental testing}

\subsection{Test procedure}

A structured approach was formulated to perform testing and demonstration of the realised RIMCAW climbing robot. Three tests were planned to verify the integrity of key aspects of the design; if there was failure of one, the remaining tests could not proceed. These tests are as follows:

- Test 1: to assess the neutral buoyancy. This was performed by slowly winching the robot into the water tank and verifying that the weight of the platform was cancelled out in water via the attached floatation foam

- Test 2: to assess the water ingress integrity of the robot; after complete submersion of the platform, all the electronic components and devices had to be confirmed to be fully functioning, and

- Test 3: to assess the functionality of the mobile robot, verifying the crawler's ability to climb the test chain.

The command station was set up next to the water tank at TWI Middlesbrough, UK and a split screen display was set up to view the video from Figure 10. Compressed air was connected to the enclosures via a regulator, set to 0.3 Bar. Using winch straps connected to the eyebolts of the platform, the gantry system 
for the test facility was used to pick up and slowly lower the RIMCAW robot into the water tank.

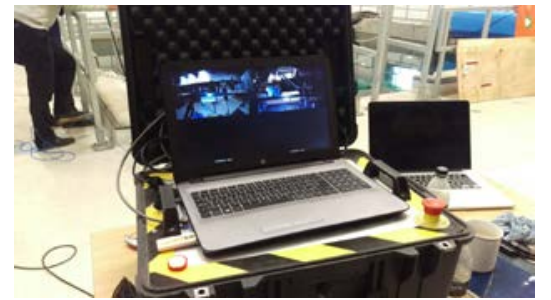

a. Command station

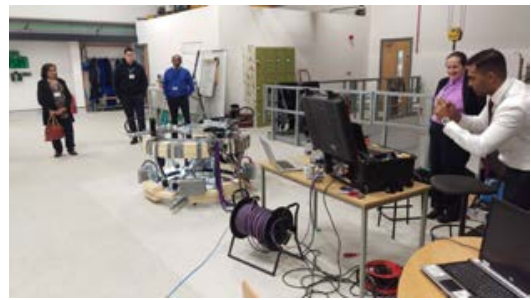

b. Testing and preparation area

Figure 10 - Testing area and command station deployed at TWI Middlesbrough

\subsection{Test 1 - Neutral buoyancy}

For this test the robot was slowly submerged using the site gantry system over the tank. The operators of the gantry were instructed not to provide too much slack or move the robot too quickly. At a certain point during the lowering process, the winch straps became slack confirming buoyancy of the system. The buoyancy was able to fully counteract the weight of the robot. However, it was noted that part of the platform remained slightly above the water line, indicating that the robot had positive buoyancy. This was deemed to be acceptable as the buoyancy could be adjusted during the testing process if it was found to affect the climbing functionality.

\subsection{Test 2 - Water ingress integrity}

The robot was submerged for $\approx 15$ minutes, to allow for all trapped air bubbles to escape. Air leaks are indicated by a visible streams of air bubbles, which were then searched for. The seals on all the enclosure lids were found to be satisfactory. Minor leaks were found to be coming from the cable glands on the gripper control box indicating it was not tightened properly. Proceeding with Test 2 was deemed to be satisfactory provided that compressed air was connected to maintain the positive pressure in the enclosures for maintaining water ingress integrity. While still in the water, the functionality of sensors and actuators was checked. The Stewart platform and manipulators were moved and were verified to be functioning correctly.

\subsection{Test 3 - Chain climbing functionality}

The mooring chain was introduced into the tank before the robot was lowered next to it. Two overhead gantry systems could not bring their end effectors together, 
requiring the platform to be lowered into the tank, with sufficient slack on the winch to allow the diver to move the robot onto the mooring the chain, Figure 11.

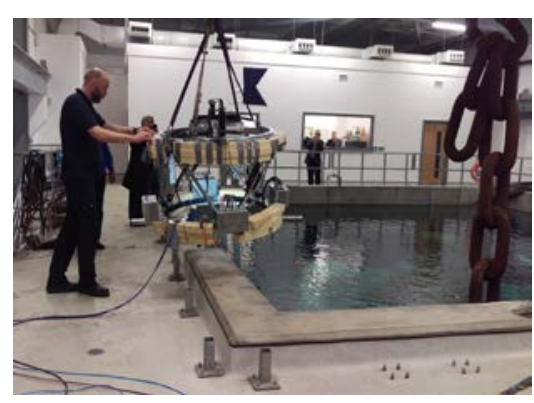

1: Robot raised for moving to water tank

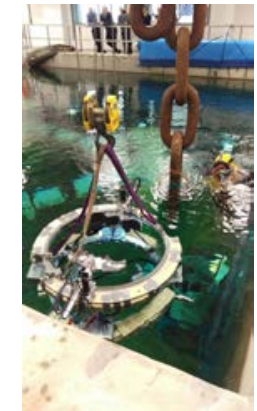

2: Robot into water tank

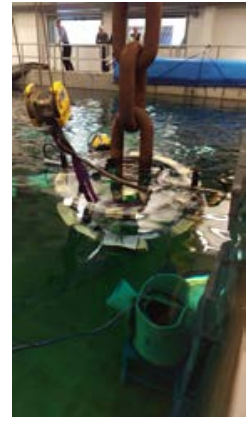

3: Robot onto chain

Figure 11 - Deployment of RIMCAW robot prototype platform

This was executed without any problems; one diver only was able to successfully manoeuvre the latch-opened robot around the chain, close and lock the latches as shown in Figure 12. However, when it came to grasping the mooring chain, the grippers could not reach the chain. This was found to be an electrical limitation preventing the actuators from achieving full extension.

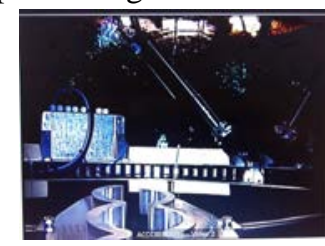

a. Submerged robot

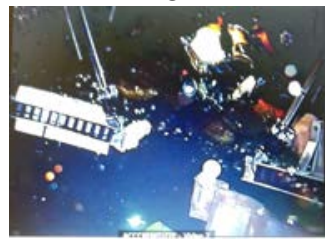

b. Diver opening latch

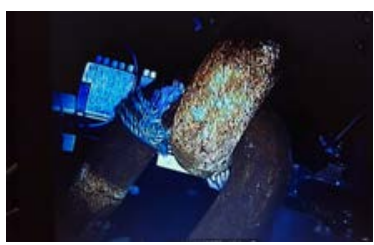

c. Robot placed onto the chain

Figure 12 - On-board camera view of the attachment to the mooring chain

\section{Improvements and result analysis}

Unfortunately, the effectiveness of the chain climbing strategy was unable to be assessed. During the detailed design phase, modelling and basic motion simulations were created to understand the dynamics of the prototype platform better. However, Test 3 was key to highlighting the successes and flaws in the system. Through preliminary experimentation and testing, minor improvements were noted to improve the robot's ability to climb.

The overall weight was higher than calculated and the gripper design, specifically, was a lot heavier than it needed to be. This in turn had a systemic effect on the rest of the system but it did not hinder the in-water tests but limited the robot's 
capabilities in the air. The actuators chosen for the Stewart platform struggled to lift the total mass of the robot in the air. Similarly, the grippers and associated actuated mechanisms were not designed to support the resulting load. This would have caused a dangerously large moment being applied to the gripper linear actuator fixtures on the top and bottom frames. In addition to reducing the weight of the overall robot, the gripping assembly would require further refinement and reinforce to handle the stresses of climbing in the air and the FPSO splash zone water forces.

\section{Conclusions and future work}

Several technical challenges and problems were experienced during the design and testing phases. Minor software and electronic problems prevented completion of the final functionality tests. Although NDT inspection testing of mooring chains was not completed, the developed RIMCAW robot met the initial design specifications for mobility and can operate underwater on mooring chains as required. The robot is being further developed to meet different underwater inspections applications. The aim is to make improvements to facilitate developments and testing so that commercially viable means of inspecting underwater assets is possible.

RobotX is an Innovate UK project that's objective is develop an autonomous flexible riser climbing platform. Due to the in-operation nature of flexible subsea risers, similar traversing challenges are faced with mooring chains, such as catenary curves, tidal waves and currents. From the lessons learnt developing the RIMCAW platform, several innovations hope to be incorporated and enhanced during the development of this project. Which would lead to a market ready universal product that could traverse both risers and mooring chains.

\section{Acknowledgments}

This research was supported by Innovate UK under the RIMCAW project. The authors are grateful to all other partners from CIT and TWI involved in the project.

\section{References}

1. $\quad$ N. D. E. Limited, "Floating production system -JIP FPS mooring integrity," Health and Safety Executive, Aberdeen, 2016.

2. M. Kai-tung, A. Duggal, P. Smedley, D. L'Hostis and S. Hongbo, "A Historical review on Integrity issues of permanent mooring systems," in Houston, Texas, Offshore Technology Conference, 2013. 
3. P. Elman, J. Bramande, E. Elletson and K. Pinheiro, "Reducing Uncertainty Through the Use of Mooring Line Monitoring," in Offshore Technology Conference, Rio de Janeiro, Brazil, 2013.

4. T.P. Sattar, A.Ruiz Garcia, M. Sanz Correa, M., \& B.S. Filloy Rodriguez , Inspection of floating platform mooring chains with a climbing robot. 17th International Conference on Climbing and Walking Robots and the Support Technologies for Mobile Machines (CLAWAR 2014), Poland.

5. S. Williams, "cordis.europa.eu," 14 March 2008. [Online]. Available: http://cordis.europa.eu/docs/publications/1216/121625181-6_en.pdf. [Accessed 10 Dec 2015].

6. Design and Prototype of a Magnetic Adhesion Tracked-Wheel Robotic Platform for Mooring Chain Inspection, Mahesh Dissanayake, Tariq Sattar, Tat-Hean Gan, Ivan Pinson, Sheehan, Lowe, Proc. Institution of Mechanical Engineering Part 1: Journal of Systems and Control Engineering. sagepub.co.uk/journals Permissions. nav DOI: 10.1177/0959651818774479 journals.sagepub.com/home/pii

7. Mahesh Dissanayake, Tariq P. Sattar, Tat-Hean Gan, Ivan Pinson (2017) Tracked -Wheel Crawler Robot for Vertically Aligned Mooring Chain Climbing, for ICIIS'2017 in Robotics, Control and Automation track

8. Mahesh Dissanayake, Tariq Sattar, Tat-hean Gan, Ivan Pinson, Shehan Lowe (2018) Adaptable legged-magnetic adhesion tracked wheel robotic platform for misaligned mooring chain climbing and inspection, Industrial Robot, Accepted for publication.

9. Elman, P., Bramande, J., Elletson, E., \& Pinheiro, K. (2013, October 29). Reducing Uncertainty Through the Use of Mooring Line Monitoring. Offshore Technology Conference. doi:10.4043/24388-MS 